\title{
De novo sequencing and analysis of the transcriptome of Panax ginseng in the leaf-expansion period
}

\author{
SHICHAO LIU ${ }^{1}$, SIMING WANG ${ }^{1}$, MEICHEN LIU ${ }^{1}$, FEI YANG ${ }^{2}$, \\ HUI ZHANG ${ }^{1}$, SHIYANG LIU ${ }^{3}$, QUN WANG ${ }^{1}$ and YU ZHAO ${ }^{1}$ \\ ${ }^{1}$ Center of Chinese Medicine and Bio-Engineering Research, Changchun University of Chinese Medicine; \\ ${ }^{2}$ Department of Bio-Pharmaceutical Development, GeneScience Pharmaceuticals Co., Ltd.; \\ ${ }^{3}$ College of Life Sciences, Jilin University, Changchun, Jilin 130000, P.R. China
}

Received July 14, 2015; Accepted May 9, 2016

DOI: $10.3892 / \mathrm{mmr} .2016 .5376$

\begin{abstract}
Panax ginseng, a traditional Chinese medicine, is used worldwide for its variety of health benefits and its treatment efficacy. However, it is difficult to cultivate due to its vulnerability to environmental stresses. The present study provided the first report, to the best of our knowledge, of transcriptome analysis of ginseng at the leaf-expansion stage. Using the Illumina sequencing platform, $>40,000,000$ high-quality paired-end reads were obtained and assembled into 100,533 unique sequences. When the sequences were searched against the publicly available National Center for Biotechnology Information protein database using The Basic Local Alignment Search Tool, 61,599 sequences exhibited similarity to known proteins. Functional annotation and classification, including use of the Gene Ontology, Clusters of Orthologous Groups, and Kyoto Encyclopedia of Genes and Genomes databases, revealed that the activated genes in ginseng were predominantly ribonuclease-like storage genes, environmental stress genes, pathogenesis-related genes and other antioxidant genes. A number of candidate genes in environmental stress-associated pathways were also identified. These novel data provide useful information on the growth and development stages of ginseng, and serve as an important public information platform for further understanding of the molecular mechanisms and functional genomics of ginseng.
\end{abstract}

Correspondence to: Professor Yu Zhao, Center of Chinese Medicine and Bio-Engineering Research, Changchun University of Chinese Medicine, 1035 Boshuo Street, Changchun, Jilin 130000, P.R. China

E-mail: cnzhaoyu1972@126.com

Key words: Panax ginseng, leaf-expansion period, transcriptome, environmental stress

\section{Introduction}

Panax ginseng C.A. Meyer is a perennial herb from the Araliaceae family, and its roots have been widely used in China for thousands of years as a tonic or adaptogen as it stated to promote vitality, enhance physical performance, and increase resistance to stress and aging (1-3). The annual growth cycle of ginseng is a process that begins with bud breakage and leaf expansion in the spring, followed by flowering, fruit set, ripening, leaf loss and root growth following fruiting in the autumn (4). From a root growing perspective, each step in the process is vital in the development of ginseng, particularly in the leaf-expansion period, as growth during this period determines future quality and yield $(5,6)$.

Ginseng grows well in semi-shade conditions due to its sensitivity to high temperatures. Usually, it takes 5-6 years for ginseng to grow a mature root under optimal conditions. However, ginseng cultivation is difficult due to its vulnerability to environmental stresses $(7,8)$. During long-term cultivation, plants may be attacked by several abiotic and fungal pathogens, which can cause severe damage to the plants $(9,10)$. To survive under different stresses, plants have developed mechanisms to perceive external signals and manifest adaptive responses, which are accompanied by appropriate physiological changes (11). These clues can assist in identifying specific unknown genes in ginseng.

Transcriptome analysis is useful and convenient for the identification of novel genes, and provides information on gene expression, gene regulation and the amino acid content of proteins (12-14). Although RNA sequencing-based transcriptome analysis of ginseng has been performed, its transcriptome has not been comprehensively described, and the stress-associated genes active during growth remain to be fully elucidated $(15,16)$.

In the present study, using Illumina technology, $>3,000,000,000$ bases of high-quality cDNA sequence were generated from ginseng in the leaf-expansion period without any prior genome information. Environmental stress-associated proteins, including pathogenesis-related proteins, antioxidants, metal stress proteins and other stress-induced proteins, were also found for the first time. The assembled, annotated transcriptome sequences and gene expression 
profiles not only provide valuable information closely associated with its medicinal effects, but are also important for further investigations on gene identification, variety selection, quality control, genetic resource development and secondary metabolites.

\section{Materials and methods}

Plant materials. Panax ginseng roots in the leaf-expansion period were used as experimental materials in the present study. The samples were collected from Fusong County (Jilin, China). The fresh tissues were cut into small sections of $>1 \mathrm{~cm}$ following cleaning, and these were immediately frozen in liquid nitrogen and stored in $-80^{\circ} \mathrm{C}$ freezers until use.

RNA and library preparation for transcriptome analysis. The samples were homogenized to a fine powder in liquid nitrogen and total RNA was isolated using the improved method with TRIzol reagent (Invitrogen; Thermo Fisher Scientific, Inc., Waltham, MA, USA), according to the manufacturer's protocol. The RNA quality was analyzed using an ethidium bromide-stained 1\% agarose gel, and RNA integrity was confirmed using an Agilent 2100 Bioanalyzer (Agilent Technologies, Inc., Santa Clara, CA, USA) $(17,18)$. The total RNA for transcriptome analysis was purified using an Illumina kit (Takara Biotechnology, Co., Ltd., Dalian, China), according to the manufacturer's protocol. The mRNA was purified using oligo (dT) magnetic beads, and then randomly segmented into small fragments using divalent cations in a fragmentation buffer (Takara Biotechnology, Co., Ltd.) (19). The cleaved RNA fragments were used as templates to synthesize first-strand cDNA using random hexamer primers. Second-strand cDNA was synthesized using ribonuclease (RNase)H (Takara Biotechnology, Co., Ltd.) and DNA polymerase I (Takara Biotechnology, Co., Ltd.) (20). The short fragments were purified using a polymerase chain reaction (PCR) extraction kit (Takara Biotechnology, Co., Ltd.) and connected with sequencing adapters (Illumina, Inc., San Diego, CA, USA). Following agarose gel electrophoresis, suitable fragments $(250 \pm 25 \mathrm{bp})$ were selected for PCR amplification as templates.

Sequencing, de novo assembly and functional annotation. Library sequencing was performed on the Illumina sequencing platform (HiSeq 2000) (21). The average fragment size of the library was $200 \mathrm{bp}$, and both ends of the fragments were sequenced (22). The raw reads were cleaned by removing the adaptor sequences, empty reads and low-quality sequences (reads with unknown sequences; 'N'). These raw reads were randomly clipped into 29 -mers for sequence assembly using Trinity software version 2.0 (github. com/trinityrnaseq/trinityrnaseq/wiki) (23). Small K-mers resulted in graphic outputs, which were too complex to be meaningful, whereas large K-mers resulted in poor overlap in regions with low sequencing depth (24). The contigs were subjected to further processing by unigene clustering to form longer sequences without $\mathrm{N}$. To assemble all the unigenes from the two samples and form a single set of non-redundant unigenes, the unigenes were clustered using TIGR Gene Indices Clustering (TGICL) tools (25). Quantification of the transcript levels were in reads per kilobase of exon mode per million mapped reads (RPKM) (26).

Unigenes are firstly aligned by blastx to protein databases of $\mathrm{Nr}$ (ftp://ftp.ncbi.nih.gov/blast/db/FASTA) and Swiss-Prot database (www.uniprot.org/). Blast2Go software (www. blast2go.com) was used to obtain functional annotations using Gene Ontology terms (GO; geneontology.org) $(27,28)$. The Clusters of Orthologous Groups (COG) database (www. ncbi.nlm.nih.gov/COG) was also used to predict and classify the functions of the unigenes, and the Kyoto Encyclopedia of Genes and Genomes (KEGG) pathway database (www. genome.jp/kegg/kegg1.html) was used to perform GO functional classification and a survey of the biological pathways of the unigenes $(29,30)$.

Reverse transcription-quantitative (RT-q)PCR validation. The selected genes, which were identified in the transcriptome sequencing analysis, were validated and quantified using RT-qPCR. Primers were designed according to the Illumina sequencing data with PrimerPremier 5.0 (www.premierbiosoft.com/primerdesign/index.html ). The quantitative reaction was performed using an Mx3000p Real-Time PCR detection system with the One Step SYBR PrimeScript PLUS RT-PCR kit (Takara Biotechnology Co., Ltd.). The PCR amplification was performed in a $25 \mu \mathrm{l}$ mixture containing $2 \mu \mathrm{l} \mathrm{cDNA}$, $0.5 \mu \mathrm{l}$ each primer, 12.5 $\mu \mathrm{l}$ SYBR Premix Ex Taq, $0.5 \mu 1$ ROX reference dye II and $9 \mu \mathrm{l}$ distilled water. The reaction was performed under the following thermocycling conditions: Initial denaturation, $95^{\circ} \mathrm{C}$ for $30 \mathrm{sec} ; 40$ cycles of denaturation at $95^{\circ} \mathrm{C}$ for $5 \mathrm{sec}$, annealing at $54^{\circ} \mathrm{C}$ for $15 \mathrm{sec}$ and extension at $72^{\circ} \mathrm{C}$ for $30 \mathrm{sec}$. The relative expression levels were calculated by comparing the quantification cycle $(\mathrm{Cq})$ value of the target gene with that of the housekeeping gene, TH-t (data not shown). Relative gene expression levels were calculated using the $2^{-\Delta \Delta \mathrm{Cq}}$ method (31). All the primer sequences used for RT-qPCR are listed in Table I.

\section{Results}

Illumina sequencing, assembly and sequence analysis. To develop a comprehensive overview of the ginseng transcriptome in the leaf-expansion period, total RNA was extracted from ginseng roots and reverse transcribed into cDNA. Following cleaning and quality checks, 40,385,232 high-quality reads were obtained, with an average length of 90 bp. The data were deposited in the National Center for Biotechnology Information (NCBI) ArrayExpress repository under the accession number E-MTAB-974. The raw reads, which were clipped into 29-mers, were assembled into 161,176 contigs with a mean length of $277 \mathrm{bp}$. Using paired-end joining and gap-filling, the contigs were further assembled into unique sequences using Trinity software. Following clustering using TGICL software, 100,533 unigenes were produced, with a mean length of $452 \mathrm{bp}$ (Table II). The length distribution of the contigs is shown in Fig. 1A, and of that of the unigenes is shown in Fig. 1B. The results showed that $>41,236$ unigenes $(41.01 \%)$ were $>500 \mathrm{bp}$ in length.

Functional annotation of the transcriptome. A sequence similarity search was performed against the NCBI non-redundant 
Table I. Primer sequences used for reverse transcription-quantitative polymerase chain reaction analysis.

\begin{tabular}{lll}
\hline \multicolumn{1}{c}{ No. } & \multicolumn{1}{c}{ Gene } & \\
\hline 0 & Thioredoxin H-type & F: CCGAAGAAGGACAGGTGATTAG \\
& & R: GGGTAATGAAACGGCAAGG \\
1 & Ethylene-forming-enzyme-like dioxygenase & F: CAGGACAAACAAGTGGAAGG \\
& & R: AACCCTGTGTAACGGGCTCT \\
2 & Nodulin MtN3 family protein & F: TTCTGCCTGGTATGGTTTGC \\
3 & Actin 7 & R: GTGAGAATGAAGGAGAGGAGTC \\
4 & & F: GTGAAGGCTGGCTTTGCTG \\
& Cytokinin-regulated kinase 1 & R: GGATGCTCTTCAGGGGCAACAC \\
5 & & F: TTGGGAGTGGAAGTTTTGGT \\
& R: AATGCGTTCTTGTTGTCGTT \\
6 & Phytochrome & F: AGACATCAAGTTCAGGCACC \\
& & R: TGACGAAACAGATGTAGGGC \\
& & F: TTCTGCCTGGTATGGTTTGC \\
& & R: GTGAGAATGAAGGAGAGGAGTC
\end{tabular}

F, forward; R, reverse.

Table II. Overview of the sequencing and assembly.

\begin{tabular}{lc}
\hline Feature & Statistic \\
\hline Total number of reads (n) & $40,385,232$ \\
Total base pairs (bp) & $3,634,670,880$ \\
Average read length (bp) & 90 \\
Total number of contigs (n) & 161,176 \\
Mean length of contigs (bp) & 277 \\
Total number of unigenes (n) & 100,533 \\
Mean length of unigenes (bp) & 452 \\
\hline
\end{tabular}

(nr) databases and Swiss-Prot protein database. There were 61,599 gene matches (61.27\% of all distinct sequences) in the nr database, and 39,506 protein matches $(39.30 \%$ of all distinct sequences) in the Swiss-Prot database.

GO assignments based on sequence homology were used to classify the functions of the distinct sequences; the 30,181 sequences in the library were categorized into 44 functional groups (Fig. 2). In the three major categories (biological process, cellular component and molecular function), 55,395, 61,279 and 31,495 GO terms were assigned, respectively. It was noted the majority of the unigenes were annotated with 'metabolic process' (12,814 members; $23.13 \%)$, 'cellular process' (12,520 members; 22.60\%), and 'response to stimulus' (4,796 members; $8.66 \%)$ under the 'biological process' category. By contrast, few genes were found in the clusters 'rhythmic process' (seven members), 'cell killing' (four members), 'extracellular region part' (four members), 'nitrogen utilization' (two members), and 'translation regulator activity' (two members). In the 'cellular component' category, unigenes annotated with 'cell' (20,899 members; $34.10 \%)$, 'cell part' (19,034 members; 31.06\%), and 'organelle'
(14,991 members; $24.46 \%)$ were predominant. The 'catalytic activity' (14,344 members; $45.54 \%)$ and 'binding' (14,014 members; $44.50 \%$ ) classes were the most abundant in the 'molecular function' category.

To evaluate the completeness of the transcriptome library produced in the present study and the effectiveness of the annotation process, all unigenes were subjected to a search against the COG database. From 61,599 nr hits, 27,232 genes were clustered into 25 function categories (Fig. 3). The 'general function prediction only' cluster was found to be the major COG category (4,306 members; $15.81 \%)$, followed by 'transcription' (2,352 members; $8.64 \%)$, 'posttranslational modification, protein turnover, chaperones' $(2,264$ members; 8.31\%), and 'replication, recombination and repair' (2,113 members; $7.76 \%)$. The 'nuclear structure' (13 members) and 'extracellular structures' (five members) categories were the least-represented groups.

To identify the biological pathways active during the ginseng growth stage investigated, the annotated sequences were also mapped to the KEGG database. As a result, 24,486 sequences were assigned to 121 KEGG pathways. The pathways with the most representation by unique sequences were metabolic pathways (18.28\%), followed by those associated with biosynthesis of secondary metabolites (8.95\%) and plant-pathogen interaction (5.78\%; Fig. 4). These annotations provide a valuable resource for investigating specific processes, functions and pathways in ginseng.

Candidate genes involved in environmental stress. The top 10 most abundant transcripts in the ginseng transcriptome library are shown in Table III, with ribonuclease-like storage protein being the most abundant. A number of environmental stress genes were found among the transcripts in the library, including pathogenesis-related, antioxidant-related and metal stress proteins. There were several pathogenesis-related 
A

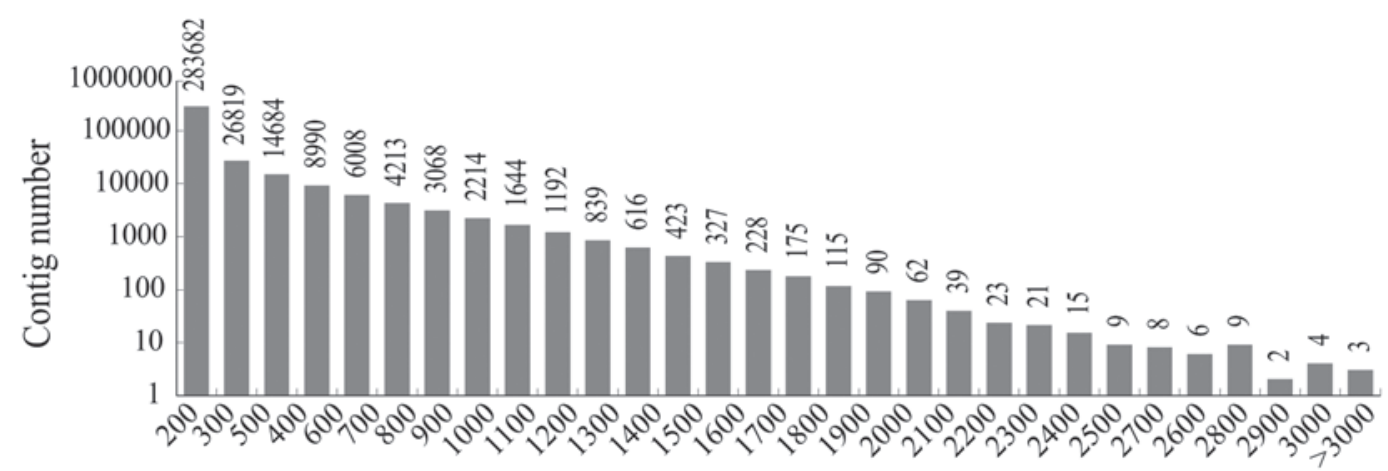

B

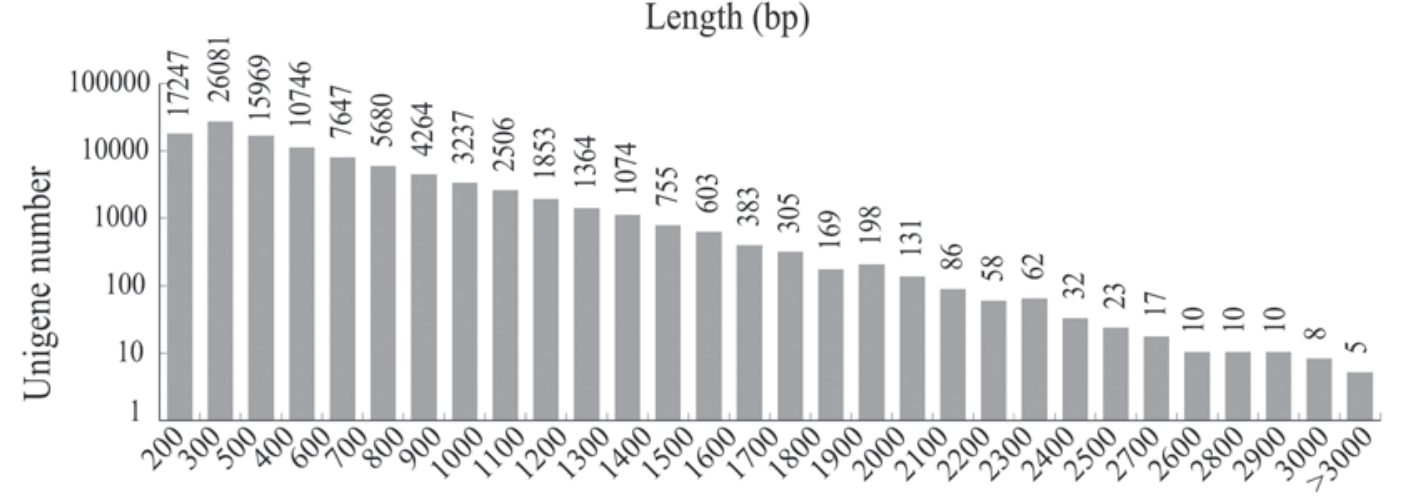

Length (bp)

Figure 1. Overview of the ginseng transcriptome assembly. (A) Size distribution of contigs. (B) Size distribution of unigenes.

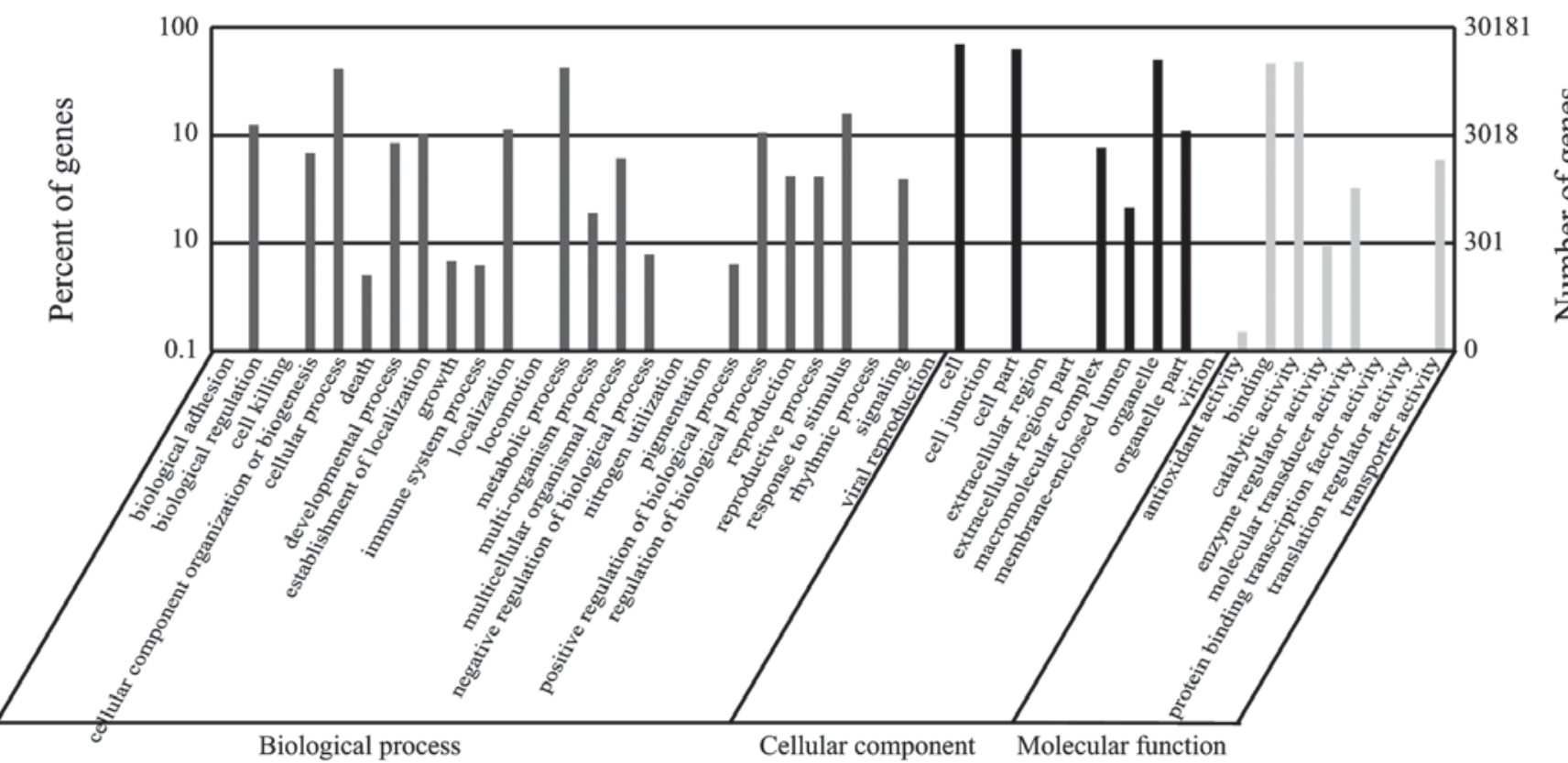

Figure 2. Functional annotation based on Gene Ontology categorization. The right $y$-axis indicates the number of genes in a category; the left y-axis indicates the percentage of a specific category of genes in that main category.

proteins (PRs) in the data set, including PR1, PR2, PR4, PR5 and PR10, with PR10 having the highest transcript level. A number of antioxidant genes expressed at high levels, including catalase and superoxide dismutase, were found in the present study (Tables IV and V). The present study also analyzed metal stress proteins, heat shock proteins (HSPs), salt stress proteins and water stress proteins, which may be involved in plant defense responses (Table VI). The accumulation of stress tolerance and pathogen response proteins in ginseng roots showed that it was sensitive to external environmental conditions in the leaf-expansion period.

$R T$ - $q P C R$ validation. The accuracy of the data obtained in the present study for several genes was assessed by examining 


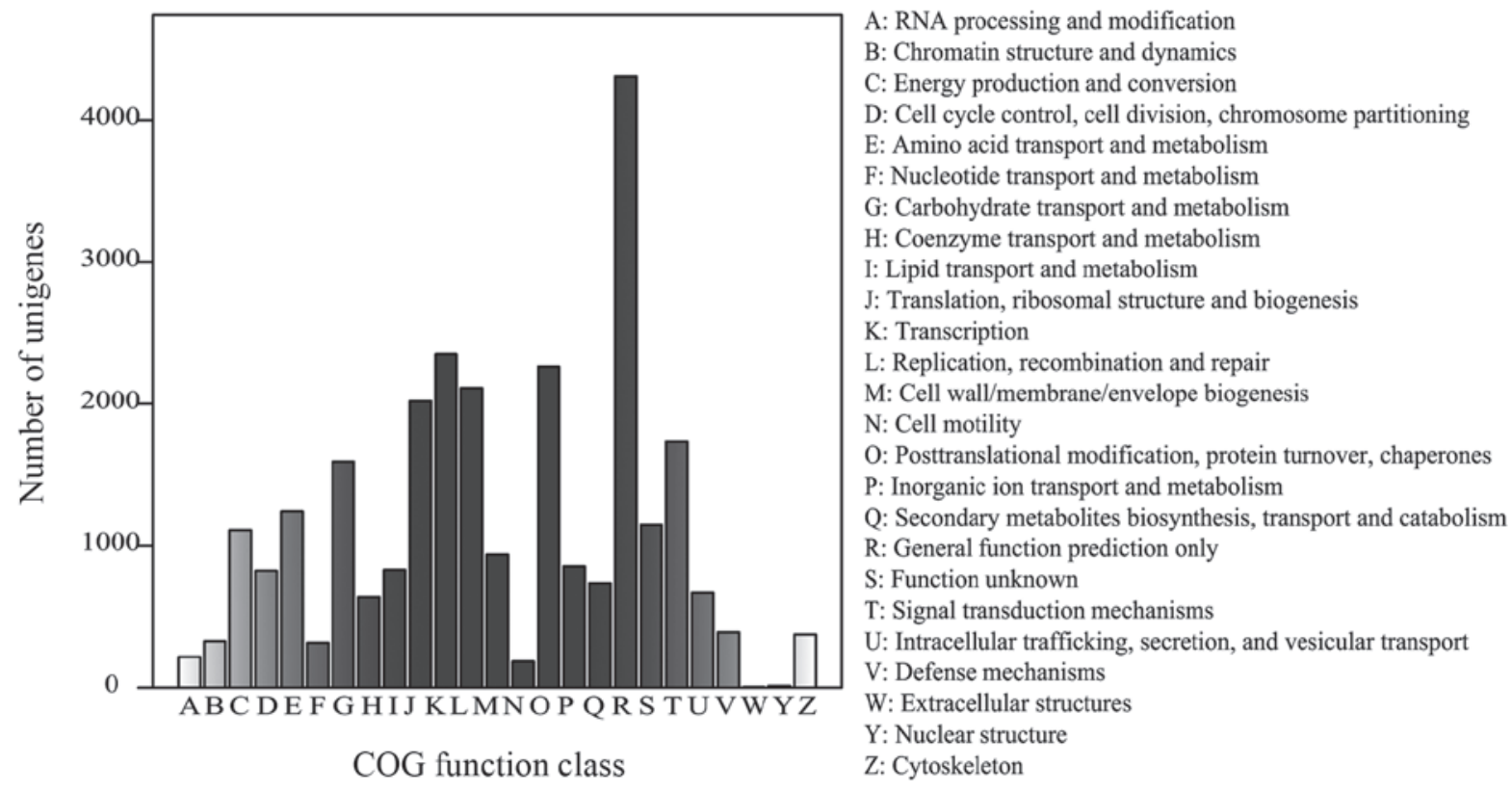

Figure 3. Classification of the clusters of orthologous groups.

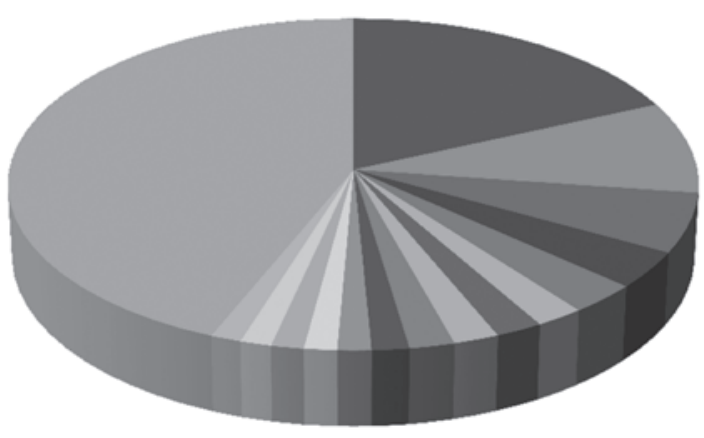

Metabolic pathways[5629]

Biosynthesis of secondary metabolites[2756]

Plant-pathogen interaction[1779]

Spliceosome[1111]

Protein processing in endoplasmic reticulum[915]

- Starch and sucrose metabolism[644]

Ribosome[626]

Purine metabolism[606]

Ubiquitin mediated proteolysis[601]

- Pyrimidine metabolism[494]

Oxidative phosphorylation[448]

RNA degradation[436]

Endocytosis[432]

Glycolysis/Gluconeogenesis[415]

Amino sugar and nucleotide sugar metabolism[408]

Other[13491]

Figure 4. Kyoto Encyclopedia of Genes and Genomes database biochemical mapping for ginseng.

their expression levels using RT-qPCR analysis. The RT-qPCR results were in accordance with the RPKM values of the respective genes. The RPKM values showed a progressive decrease, and the $2^{-\Delta \Delta \mathrm{Cq}}$ values demonstrated a similar trend, as shown in Fig. 5.

\section{Discussion}

Illumina sequencing technology offers novel potential in high-throughput sequencing for the majority of species, as it provides an accurate, rapid and cost-effective method for transcriptome analysis (32). In the present study, ginseng in the leaf-expansion period was analyzed using this technology. A draft sequence was successfully generated and assembled, and high sensitivity in the coverage of weakly expressed genes was found. Additionally, the present study provided a basis for the functional analysis of genes involved in ginseng development without prior genome annotation, and demonstrated the feasibility of using the sequencing-based Illumina system for gene expression profiling.

Prior to the present study, ginseng was represented by only 565 sequences in the NCBI protein database and 12,071 sequences in the NCBI Expressed Sequence Tags database. In the present study, $>40,000,000$ reads with a mean length of $90 \mathrm{bp}$ were produced. The high-quality reads were assembled into 100,533 unique sequences with a mean length of $452 \mathrm{bp}$, and 61,599 (61.27\%) sequences showed a BLAST match above the cut-off level. These findings represent a substantial contribution to the existing sequence resources for ginseng and facilitate the acceleration of investigations into pharmacologically active substances.

To evaluate the completeness of the transcriptome library produced in the present study, and the effectiveness of the annotation process, the sequences were annotated using GO classifications, COG classifications and KEGG pathways. In total, 30,181 sequences were assigned to $44 \mathrm{GO}$ classifications, 
Table III. Transcripts expressed at high levels in the ginseng library.

\begin{tabular}{cllr}
\hline No. & \multicolumn{1}{c}{ ID } & \multicolumn{1}{c}{ Non-redundant annotation } & RPKM \\
\hline 1 & Unigene48956_F1 & Ribonuclease-like storage protein & $105,492.10$ \\
2 & Unigene49972_F1 & Pathogensis-related protein 10 (Panax ginseng) & $22,469.66$ \\
3 & Unigene48226_F1 & Metallothionein-like MT-3 (Jatropha curcas) & $16,573.52$ \\
4 & Unigene49842_F1 & Specific tissue protein 2 (Cicer arietinum) & $10,068.34$ \\
5 & Unigene49004_F1 & Basic blue copper protein (Cicer arietinum) & $8,531.23$ \\
6 & Unigene47883_F1 & Kirola allergen & $8,292.52$ \\
7 & Unigene16230_F1 & Hypothetical protein (Vitis vinifera) & $6,682.50$ \\
8 & Unigene48659_F1 & Catalase (Solanum tuberosum) & $6,231.77$ \\
9 & Unigene49760_F1 & Phloem protein 2-2 (Apium graveolens dulce group) & $4,874.98$ \\
10 & Unigene48454_F1 & $\beta$-amylase (Castanea crenata) & $4,849.63$
\end{tabular}

RPKM, reads per kilobase of exon mode per million mapped reads.

Table IV. Expressed transcripts of pathogenesis-related proteins in the ginseng library.

\begin{tabular}{lllr}
\hline No. & \multicolumn{1}{c}{ ID } & \multicolumn{1}{c}{ Non-redundant annotation } & RPKM \\
\hline 1 & Unigene49972_F1 & Pathogensis-related protein 10 (Panax ginseng) & $22,469.66$ \\
2 & Unigene22713_F1 & Pathogenesis-related protein 1 (Vitis hybrid cultivar) & $1,264.81$ \\
3 & Unigene48216_F1 & Pathogenesis-related protein (Lepidium latifolium) & 654.54 \\
4 & Unigene49556_F1 & Pathogenesis-related protein PR-4 type (Sambucus nigra) & 481.79 \\
5 & Unigene47993_F1 & Pathogenesis-related protein 2 (Petroselinum) & 317.11 \\
6 & Unigene41124_F1 & Pathogenesis-related protein PR10a (Nicotiana tabacum) & 15.70 \\
7 & Unigene17748_F & Pathogenesis-related protein 5 (Panax ginseng) & 2.49 \\
\hline
\end{tabular}

RPKM, reads per kilobase of exon mode per million mapped reads.

Table V. Antioxidant-associated transcripts expressed in the ginseng library.

\begin{tabular}{lllr}
\hline No. & \multicolumn{1}{c}{ ID } & \multicolumn{1}{c}{ Non-redundant annotation } & RPKM \\
\hline 1 & Unigene49614_F1 & Ascorbate peroxidase $($ Nicotiana tabacum) & $8,383.00$ \\
2 & Unigene48959_F1 & Superoxide dismutase $(\mathrm{Cu}-\mathrm{Zn})$ & $1,812.32$ \\
3 & Unigene48521_F1 & Superoxide dismutase $(\mathrm{Mn})$ & 155.30 \\
4 & Unigene8659_F1 & Chloroplast iron superoxide dismutase (Dimocarpus longan) & 3.73 \\
5 & Unigene48659_F1 & Catalase (Solanum tuberosum) & $6,231.77$ \\
6 & Unigene49928_F1 & Glutathione peroxidase 1 (Arachis hypogaea) & $1,049.05$ \\
\hline
\end{tabular}

RPKM, reads per kilobase of exon mode per million mapped reads.

16,371 sequences to $25 \mathrm{COG}$ classifications and 24,486 sequences to $121 \mathrm{KEGG}$ pathways. These annotations provide a valuable resource for investigating specific processes, functions and pathways in ginseng, and facilitate comparisons of transcript levels within and between samples. The expression levels of the genes in the data were quantified by counting the number of RPKM. The RPKM measure of read density reflects the molar concentration of a transcript in the original sample by normalizing for RNA length and the total number of reads in the measurement. This calculation normalizes the read density measurement and, therefore, can be used for comparisons within and between tissue samples (33).

Based on the de novo sequencing and analysis of ginseng roots, the present study identified the top 10 most frequent transcripts in the ginseng transcriptome library (Table III). The most abundant ginseng transcript (RPKM=10,5492.1408) was annotated as a ribonuclease-like storage protein. Of note, this type of protein has been identified as the most abundant root protein of ginseng in comparative proteome analysis (34). The present study hypothesized that the ribonuclease-like 
Table VI. Genes involved in plant defense responses in the ginseng library.

\begin{tabular}{|c|c|c|c|}
\hline Category & ID & Non-redundant annotation & RPKM \\
\hline \multirow[t]{2}{*}{ Metal stress protein } & Unigene48226_F1 & Metallothionein-like MT-3 (Jatropha curcas) & $16,573.52$ \\
\hline & Unigene48880_F1 & Metallothionein type 2 (Sesbania drummondii) & $4,290.07$ \\
\hline \multirow[t]{6}{*}{ Heat shock protein } & Unigene49007_F1 & Heat shock protein 90 (Nicotiana tabacum) & 959.88 \\
\hline & Unigene11322_F1 & Heat shock protein 70 (Arabidopsis thaliana) & 682.67 \\
\hline & Unigene492_F1 & $\begin{array}{l}\text { Class II small heat shock protein Le-HSP17.6 } \\
\text { (Solanum lycopersicum) }\end{array}$ & 161.86 \\
\hline & Unigene34233_F1 & Small molecular heat shock protein 10 & \\
\hline & & (Nelumbo nucifera) & 133.21 \\
\hline & Unigene100483_F1 & Heat shock protein 60 (Ageratina adenophora) & 12.73 \\
\hline \multirow[t]{5}{*}{ Salt stress protein } & Unigene21092_F1 & Salt tolerance protein 1 (Beta vulgaris) & 48.08 \\
\hline & Unigene5170_F1 & Salt responsive protein 2 (Solanum lycopersicum) & 141.48 \\
\hline & Unigene662_F1 & Salt tolerance protein 3 (Beta vulgaris) & 14.96 \\
\hline & Unigene6719_F1 & Salt tolerance protein 4 (Beta vulgaris) & 109.43 \\
\hline & Unigene14324_F1 & $\begin{array}{l}\text { Salt tolerance protein } 5 \text {-like protein } \\
\text { (Solanum tuberosum) }\end{array}$ & 60.38 \\
\hline \multirow[t]{10}{*}{ Water stress protein } & Unigene15597_F1 & Dehydrin 2 (Panax ginseng) & 82.85 \\
\hline & Unigene21543_F1 & Dehydrin 3 (Panax ginseng) & $1,532.60$ \\
\hline & Unigene12318_F1 & Dehydrin 4 (Panax ginseng) & 13.96 \\
\hline & Unigene36768_F1 & Dehydrin 5 (Panax ginseng) & 11.93 \\
\hline & Unigene4890_F1 & Dehydrin 6 (Panax ginseng) & 17.87 \\
\hline & Unigene1_F1 & Dehydrin 8 (Panax ginseng) & 70.96 \\
\hline & Unigene54318_F1 & Dehydrin 9 (Panax ginseng) & 1.87 \\
\hline & Unigene6458_F1 & $\begin{array}{l}\text { Lipid transfer protein-like protein } \\
\text { (Noccaea caerulescens) }\end{array}$ & 16.79 \\
\hline & Unigene5623_F1 & $\begin{array}{l}\text { Late embryogenesis abundant protein (LEA) } \\
\text { family protein (Arabidopsis thaliana) }\end{array}$ & 74.65 \\
\hline & Unigene15942_F1 & 14 kDa proline-rich protein DC2.15; Flags: Precursor & 360.01 \\
\hline
\end{tabular}

RPKM, reads per kilobase of exon mode per million mapped reads.

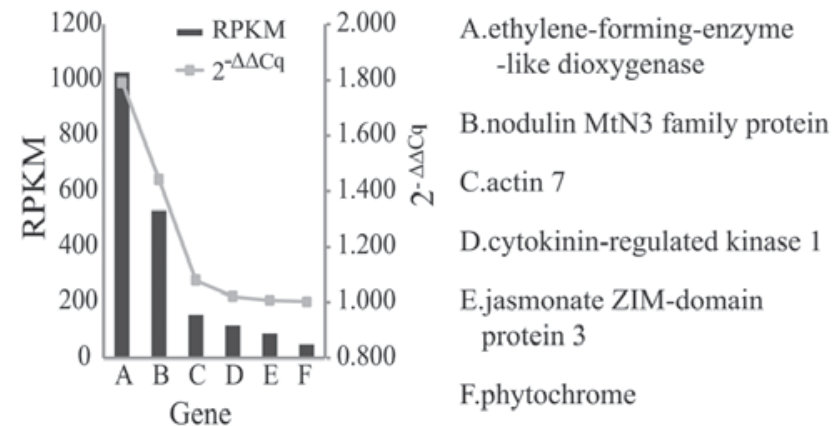

Figure 5. Results of the reverse transcription-quantitative polymerase chain reaction validation of ginseng. The right $y$-axis indicates the value of $2^{-\Delta \Delta C q}$; the left $y$-axis indicates the RPKM value. RPKM, reads per kilobase of exon mode per million mapped reads.

storage protein may accumulate in the leaf-expansion period to accompany changes in the physiological characteristics of ginseng as the aerial parts of ginseng grow rapidly during this stage, which requires a high level of nutrients. Additionally, transcripts encoding $\beta$-amylase were expressed at high levels as starch is the most abundant component of ginseng roots.
Based on the frequencies of transcripts in the ginseng transcriptome library, the present study found that several of the candidate genes associated with environment stress were expressed at high levels. To survive under different levels of stress, plants have developed mechanisms to perceive external signals and manifest adaptive responses, accompanied by appropriate physiological changes (11). One of the notable plant defense responses is the induction of PRs, which have been classified into 17 families, based on their structural differences, serological associations and biological activities $(35,36)$. In total, five groups of PRs were found in the present study, including PR1, PR2, PR4, PR5 and PR10, which have been characterized as $\beta-1,3$-glucanases, chitinases, and thaumatin-like and ribonuclease-like proteins $(37,38)$. The PR10 protein was first described in cultured parsley cells, and this protein family consists of relatively diverse members, which are sub-grouped into different functional classes (39). It has been suggested that PR10 proteins are involved in plant defense as the genes are usually induced in environmental stress and attack by various pathogens. To date, three previously isolated PR 10 proteins from ginseng have been characterized as ribonucleases (RNases), and several purified 
PR10 proteins have exhibited in vitro RNase activity $(11,40)$. Other PRs were also expressed at high levels in the present study, and high expression levels of PRs during this growth period are associated with the growth environment.

As shown in Table V, several antioxidant enzymes expressed at high levels were also identified. Catalase (CAT), the antioxidant enzyme with the highest level of expression, was shown to be abundant in the rhizome, and has also been identified in seedling shoots of 4-year-old ginseng and 11-year-old ginseng cultured in vitro (41). Superoxide dismutase, found in the mitochondrial intermembrane space, catalyzes dismutation of the superoxide radical to $\mathrm{H}_{2} \mathrm{O}_{2}$ and oxygen (42). $\mathrm{H}_{2} \mathrm{O}_{2}$ is converted to water and oxygen by catalase, ascorbate peroxidase or glutathione peroxidase in peroxisomes; glutathione peroxidase enzymes also have the ability to detoxify peroxides (43). Each of these enzymes has a physiological function in the absence of stress, and they are all increased under abiotic stress (42). CAT is significantly induced under different stresses, including heavy metals, plant hormones, osmotic agents, high light irradiance and abiotic stresses (44).

The present study found that certain metal stress proteins were expressed at high levels, including metallothionein-like MT-3 and metallothionein type 2, which have metal-chaperoning and reactive oxygen species-scavenging functions (45). Certain HSPs, including HSP90, HSP70, HSP60 and small HSPs, were also abundant in the library produced in the present study. In stressful conditions, HSPs assist in the ability of individual cells to cope with stress by maintaining important cellular processes (46). Although HSPs are active in cells under normal circumstances, their functions are important in cells under conditions of stress (47). Five types of salt-responsive proteins were expressed at high levels, including salt-responsive proteins 1-5, as well as water stress-induced proteins, including dehydrin, lipid transfer, late embryogenesis abundant and Dc2.15 proteins (48). Dehydrins, including Lea proteins, typically accumulate in low temperatures or under conditions of water-deficit, and are considered to be involved in freezing and drought tolerance in plants $(49,50)$.

Although ginseng is used worldwide, its sources are limited. It grows in the shade, and has strict temperature and water requirements; any changes in the environment affect its growth. During extended cultivation, ginseng may be attacked by several abiotic and biotic pathogens, resulting in severe damage $(51,52)$. In the present study, it was found that genes associated with environmental stress are active during ginseng growth. These genes respond to environmental changes, affecting the growth of ginseng and the accumulation of products during extended cultivation.

In conclusion, the present study performed de novo transcriptome sequencing of ginseng in the leaf-expansion period using the Illumina platform. In total, $>40,000,000$ sequencing reads were produced and assembled into 100,533 unique sequences. By performing BLAST analysis of the unigenes against public databases ( $\mathrm{Nr}$, Swiss-Prot, KEGG and $\mathrm{COG})$, functional annotations and classifications were obtained. The large number of transcriptomic sequences produced and their functional annotations provide valuable resources for molecular investigations of ginseng. In addition, several candidate genes were identified, which are potentially involved in growth and environmental stress responses; identification of this set of candidate genes is valuable for further investigations.

\section{Acknowledgements}

This study was supported by the grant from National Natural Foundation of China (grant no. 81373937), the Scientific and Technological Development Program of Jilin, China (grant no. 20140622003JC), and the Strategic Adjustment of the Economic Structure of Jilin Province to Guide the Capital Projects (grant no. 2014N155).

\section{References}

1. Briskin DP: Medicinal plants and phytomedicines. Linking plant biochemistry and physiology to human health. Plant Physiol 124: $507-514,2000$.

2. Kim YJ, Zhang D and Yang DC: Biosynthesis and biotechnological production of ginsenosides. Biotechnol Adv 33: 717-735, 2015.

3. Smith I, Williamson EM, Putnam S, Farrimond J and Whalley BJ: Effects and mechanisms of ginseng and ginsenosides on cognition. Nutr Rev 72: 319-333, 2014

4. Wu D, Austin RS, Zhou S and Brown D: The root transcriptome for North American ginseng assembled and profiled across seasonal development. BMC Genomics 14: 564, 2013.

5. Munns R, Passioura JB, Guo J, Chazen O and Cramer GR: Water relations and leaf expansion: Importance of time scale. J Exp Bot 51: 1495-1504, 2000 .

6. Wei YC, Chen CB, Li YB, et al: Ginseng development practical manual. Jilin Science and Technology Bureau. Changchun, 2010.

7. Peng Y, Lin W, Cai W and Arora R: Overexpression of a Panax ginseng tonoplast aquaporin alters salt tolerance, drought tolerance and cold acclimation ability in transgenic Arabidopsis plants. Planta 226: 729-740, 2007.

8. Pulla RK, Kim YJ, Parvin S, Shim JS, Lee JH, Kim YJ, In JG, Senthil KS and Yang DC: Isolation of S-adenosyl-L-methionine synthetase gene from Panax ginseng C.A. meyer and analysis of its response to abiotic stresses. Physiol Mol Biol Plants 15: 267-275, 2009.

9. Lee SK: Fusarium species associated with ginseng (Panax ginseng) and their role in the root-rot of ginseng plants. Res Plant Dis 10: 248-259, 2004.

10. Li Y, Liu SL, Huang XF and Ding WL: Allelopathy of ginseng root exudates on pathogens of ginseng. Acta Ecological Sinica 29: 161-167, 2009.

11. Lee OR, Pulla RK, Kim YJ, Balusamy SR and Yang DC: Expression and stress tolerance of PR10 genes from Panax ginseng C. A. Meyer. Mol Biol Rep 39: 2365-7234, 2012.

12. Gupta P, Goel R, Pathak S, Srivastava A, Singh SP, Sangwan RS, Asif $\mathrm{MH}$ and Trivedi PK: De novo assembly, functional annotation and comparative analysis of Withania somnifera leaf and root transcriptomes to identify putative genes involved in the withanolides biosynthesis. PLoS One 8: e62714, 2013.

13. Margulies M, Egholm M, Altman WE, Attiya S, Bader JS, Bemben LA, Berka J, Braverman MS, Chen YJ, Chen Z, et al: Genome sequencing in microfabricated high-density picolitre reactors. Nature 437: 376-380, 2005.

14. Wang Z, Gerstein M and Snyder M: RNA-Seq: A revolutionary tool for transcriptomics. Nat Rev Genet 10: 57-63, 2009.

15. Chen S, Luo H, Li Y, Sun Y, Wu Q, Niu Y, Song J, Lv A, Zhu Y, Sun C, et al: 454 EST analysis detects genes putatively involved in ginsenoside biosynthesis in Panax ginseng. Plant Cell Rep 30: 1593-1601, 2011.

16. Choi DW, Jung J, Ha YI, Park HW, In DS, Chung HJ and Liu JR: Analysis of transcripts in methyl jasmonate-treated ginseng hairy roots to identify genes involved in the biosynthesis of ginsenosides and other secondary metabolites. Plant Cell Rep 23: 557-566, 2005.

17. Fleige $S$ and Pfaffl MW: RNA integrity and the effect on the real-time qRT-PCR performance. Mol Aspects Med 27: 126-139, 2006.

18. Metzker ML: Sequencing technologies-the next generation. Nat Rev Genet 11: 31-46, 2010. 
19. King M, Reeve W, Van der Hoek MB, Williams N, McComb J, O'Brien PA and Hardy GE: Defining the phosphite-regulated transcriptome of the plant pathogen Phytophthora cinnamomi. Mol Genet Genomics 284: 425-435, 2010.

20. Jiang Q, Wang F, Tan HW, Li MY, Xu ZS, Tan GF and Xiong AS: De novo transcriptome assembly, gene annotation, marker development and miRNA potential target genes validation under abiotic stresses in Oenanthe javanica. Mol Genet Genomics 290: 671-683, 2015.

21. Yu M, Yu J, Gu C, Nie Y, Chen Z, Yin X and Liu Y: De novo sequencing and transcriptome analysis of Ustilaginoidea virens by using Illumina paired-end sequencing and development of simple sequence repeat markers. Gene 547: 202-210, 2014.

22. Tsanakas GF, Manioudaki ME, Economou AS and Kalaitzis $P$ De novo transcriptome analysis of petal senescence in Gardenia jasminoides Ellis. BMC Genomics 15: 554, 2014.

23. Grabherr MG, Haas BJ, Yassour M, Levin JZ, Thompson DA, Amit I, Adiconis X, Fan L, Raychowdhury R, Zeng Q, et al: Full-length transcriptome assembly from RNA-Seq data without a reference genome. Nat Biotechnol 15: 644-652, 2011.

24. Wang Z, Zhang J, Jia C, Liu J, Li Y, Yin X, Xu B and Jin Z: De novo characterization of the banana root transcriptome and analysis of gene expression under Fusarium oxysporum f. sp. Cubense tropical race 4 infection. BMC Genomics 13: 650, 2012

25. Lee Y, Tsai J, Sunkara S, Karamycheva S, Pertea G, Sultana R, Antonescu V, Chan A, Cheung F and Quackenbush J: The TIGR Gene Indices: Clustering and assembling EST and known genes and integration with eukaryotic genomes. Nucleic Acids Res 33 (Database Issue): D71-D74, 2005.

26. Mortazavi A, Williams BA, McCue K, Schaeffer L and Wold B: Mapping and quantifying mammalian transcriptomes by RNA-Seq. Nat Methods 5: 621-628, 2008.

27. Conesa A, Götz S, García-Gómez JM, Terol J, Talón M and Robles M: Blast2GO: A universal tool for annotation, visualization and analysis in functional genomics research. Bioinformatics 15: 3674-3676, 2005.

28. Harris MA, Clark J, Ireland A, Lomax J, Ashburner M, Foulger R, Eilbeck K, Lewis S, Marshall B, Mungall C, et al: The gene ontology (GO) database and informatics resource. Nucleic Acids Res 32 (Database Issue): D258-D261, 2004.

29. Kanehisa M, Goto S, Kawashima S, Okuno Y and Hattori M: The KEGG resource for deciphering the genome. Nucleic Acids Res 32 (Database Issue): D277-D280, 2004

30. Tatusov RL, Natale DA, Garkavtsev IV, Tatusova TA, Shankavaram UT, Rao BS, Kiryutin B, Galperin MY, Fedorova ND and Koonin EV: The COG database: New developments in phylogenetic classification of proteins from complete genomes. Nucleic Acids Res 29: 22-28, 2001

31. Livak KJ and Schmittgen TD: Analysis of relative gene expression data using real-time quantitative PCR and the 2(-Delta DeltaC (T)) Method. Methods 25: 402-408, 2001.

32. Hudson ME: Sequencing breakthroughs for genomic ecology and evolutionary biology. Mol Ecol Resour 8: 3-17, 2008.

33. Wagner GP, Kin K and Lynch VJ: Measurement of mRNA abundance using RNA-seq data: RPKM measure is inconsistent among samples. Theory Biosci 131: 281-285, 2012.

34. Kim SI, Kweon SM, Kim EA, Kim JY, Kim S, Yoo JS and Park YM: Characterization of RNase-like major storage protein from the ginseng root by proteomic approach. J Plant Physiol 161: $837-845,2004$
35. Sels J, Mathys J, De Coninck BM, Cammue BP and De Bolle MF: Plant pathogenesis-related (PR) proteins: A focus on PR peptides. Plant Physiol Biochem 46: 941-950, 2008.

36. Van Loon LC, Rep M and Pieterse CM: Significance of inducible defense-related proteins in infected plants. Annu Rev Phytopathol 44: 135-162, 2006.

37. Sticher L, Mauch-Mani B and Métraux JP: Systemic acquired resistance. Annu Rev Phytopathol 35: 235-270, 1997.

38. van Loon LC: Pathogenesis-related proteins. Plant Mol Biol 4: 111-116, 1985

39. Somssich IE, Schmelzer E, Kawalleck P and Hahlbrock K: Gene structure and in situ transcript localization of pathogenesis-related protein 1 in parsley. Mol Gen Genet 213: 93-98, 1988.

40. Moiseyev GP, Fedoreyeva LI, Zhuravlev YN, Yasnetskaya E, Jekel PA and Beintema JJ: Primary structures of two ribonucleases from ginseng calluses. New members of the PR-10 family of intracellular pathogenesis-related plant proteins. FEBS Lett 28: 207-210, 1997.

41. Purev M, Kim YJ, Kim MK, Pulla RK and Yang DC: Isolation of a novel catalase (Cat1) gene from Panax ginseng and analysis of the response of this gene to various stresses. Plant Physiol Biochem 48: 451-460, 2010

42. Sathiyaraj G, Lee OR, Parvin S, Khorolragchaa A, Kim YJ and Yang DC: Transcript profiling of antioxidant genes during biotic and abiotic stresses in Panax ginseng C. A. Meyer. Mol Biol Rep 38: 2761-2769, 2011.

43. Hernandez JA, Jimenez A, Mullineaux P and Sevilia F: Tolerance of pea (Pisumsativum L.) to long-term salt stress is associated with induction of antioxidant defenses. Plant Cell Environ 23: 853-862, 2000

44. Apel K and Hirt H: Reactive oxygen species: Metabolism, oxidative stress, and signal transduction. Annu Rev Plant Biol 55: 373-399, 2004

45. Brkljacić JM, Samardzić JT, Timotijević GS and Maksimović VR: Expression analysis of buckwheat (Fagopyrum esculentum Moench) metallothionein-like gene (MT3) under different stress and physiological conditions. J Plant Physiol 161: 741-746, 2004.

46. Mohamed $\mathrm{H}$ and Al-Whaibi: Plant heat-shock proteins: A mini review. Journal of King Saud University Science 23: 139-150, 2011.

47. Jung JD, Park HW, Hahn Y, Hur CG, In DS, Chung HJ, Liu JR and Choi DW: Discovery of genes for ginsenoside biosynthesis by analysis of ginseng expressed sequence tags. Plant Cell Rep 22: 224-230, 2003.

48. Ingram $\mathbf{J}$ and Bartels $\mathrm{D}$ : The molecular basis of dehydration tolerance in plants. Annu Rev Plant Physiol Plant Mol Biol 47: 377-403, 1996

49. Pockley AG and Muthana M: Heat shock proteins and allograft rejection. Contrib Nephrol 148: 122-134, 2005.

50. Xu D, Duan X, Wang B, Hong B, Ho T and Wu R: Expression of a late embryogenesis abundant protein gene, HVA1, from barley confers tolerance to water deficit and salt stress in transgenic rice. Plant physiol 110: 249-257, 1996.

51. Shen L, Xu J, Dong LL, Li XW and Chen SL: Cropping system and research strategies in Panax ginseng. Zhongguo Zhong Yao Za Zhi 40: 3367-3373, 2015 (In Chinese).

52. Liu Y, Zhao D, Liu M, Hu CY, Li Y and Ding WL: Investigation of pests and diseases occurrence and pesticides application in main producing areas of Panax ginseng. Chinese Agricultural Science Bulletin 30: 294-298, 2014. 